\section{PLOT-SIZE IN YIELD SURVEYS}

\author{
BY DR. V. G. PANSE
}

Institute of Plant Industry, Indore

$\mathrm{R}$ ECENT communications by Sukhatme ${ }^{1,2}$ and Mahalanobis $^{3}$ have established the conclusion that sample plots of a small size (25 sq. ft. or less according to Mahalanobis) give biased estimates of yield in sample surveys; but whereas Sukhatme has shown the bias to.persist even with a plot-size of 118 sq. ft., Mahalanobis has expressed the view that it becomes practically negligible for plots of size larger than 40-50 sq. ft. Their results relate to crops grown unevenly by broadcasting the seed or sowing it in irregular lines. I have studied the problem of plot-size in yield surveys on cotton, which is sown by a drill in evenly spaced rows in Central India. The results ${ }^{4}$ are summarized here, as they provide for the first time information on this question applicable to drill-sown crops.

Plots of three sizes, $1 / 20,1 / 200$ and $1 / 2,000$ acre, were compared. In crops sown in uniformly spaced rows, plot-size is defined by a specified length of a given number of contiguous rows. The plots in the present experiment were marked according to this definition, and since the actual plot-sizes differed slightly from the standard size owing to small variations in row spacing, the plot-yields were reduced to the standard size before analysis. Two plots of each size or six plots in all were laid down at random locations in the field. The experiment was done in twenty-six fields at Indore and in an equal number of fields at Government farms in the Central Provinces. In addition to the yields of the plots, yields of whole fields were accurately measured at the Government farms; but this was unfortunately not possible at Indore. Average yields of seed cotton per acre estimated from the three plot-sizes were:

\begin{tabular}{ccc} 
Plot size & \multicolumn{2}{c}{ Yield (lb. per acre) } \\
120 acre & $195 \cdot 7$ & Central Provinces \\
$1 / 200$ " & $197 \cdot 0$ & $322 \cdot 2$ \\
$1 / 2,000$, & $221 \cdot 3$ & $354 \cdot 9$ \\
& & $421 \cdot 8$
\end{tabular}

There was a gradual increase in yield per acre as the plot-size was decreased in the Central Provinces experiment. At Indore also the yield from the smallest plot was larger than the other two. A comparison between different plot-sizes did not, however, reveal significant differences in yield, though the excess of yield estimated from $1 / 2,000$-acre plots over 1/20-acre plots in the Central Provinces was almost twice its standard error. The comparison of yields estimated from sample plots with the yield obtained by harvesting the whole fields in the Central Provinces proved more conclusive, as shown below :

$$
\begin{aligned}
& \text { Comparison of plots } \\
& \text { with whole fields } \\
& 1 / 20 \text { acre plot } \\
& 1 / 200 \text { ", ", } \\
& 1 / 2,000, \text {," }
\end{aligned}
$$

$$
\begin{gathered}
\begin{array}{c}
\text { Difference in yield } \\
\text { (lb. per acre) }
\end{array} \\
26 \cdot 7 \pm 26 \cdot 5 \\
59 \cdot 4 \pm 28 \cdot 1 \\
126 \cdot 3 \pm 57 \cdot 6
\end{gathered}
$$

The yield estimated from 1/20-acre plots agreed quite well with the yield for the whole field, as the difference between the two was no more than its standard error; but the excess of the yield estimated from the other two plots over the yield from the whole field was greater than twice its standard error and clearly significant. With $1 / 2,000$-acre plots, the over-estimation was as high as $42 \cdot 7$ per cent of true yield.

Thus, not only did the smallest plot size, 1/2,000. acre or $22 \mathrm{sq}$. ft., give highly biased results, but also there was an indication of bias also with the plot size of 1/200 acre or 218 sq. ft. in the Central Provinces experiment. The latter result would seem to show that even plots of 200 sq. ft. cannot always be relied upon to give unbiased estimates of yield. The second conclusion from this experiment is that although small plots, such as those used by English and American workers for sampling drill-sown crops, may not be open to serious objection for comparative purposes, the possibility that yield estimates derived from such plots are seriously biased needs attention.

${ }^{1}$ Sukhatme, P. V., Nature, 157, 630 (1946).

${ }^{2}$ Sukhatme, P. V., Nature, 158345 (1946).

${ }^{8}$ Mahalanobis, P. C., Nature, 158, 798 (1946).

- Panse, V. G., Curr. Sci., 15, 218 (1946).

\section{FUTURE OF PAINT*}

$\mathrm{M}$ ODERN developments in plastic materials have forced the paint industry to concern itself with an ever-increasing number of film-forming substances-synthetic rubbers, resins and oils; and researches on pigments have not lagged behind. The electron microscope reveals the shape of particles too small for optical resolution. Pigment particles had been assumed to be spherical; it transpires, however, that of the pigments commonly used only carbon particles have this shape. The shape of carbon particles is independent of the material from which the pigment is prepared. Carbon smoke is different from other inorganic smokes, which, with the exception of aluminium oxide, yield crystalline particles, and the reason for this difference has not been fully explained. The carbon pigments have another interesting property, namely, that of forming chains, like beads on a string. Such an arrangement may be due to electrostatic charges; but whatever the cause, the condition probably accounts for the high electrical conductivity of carbon-pigmented paint.

Study of the forces which exist at the interface between pigment and medium has shown that they develop the plasticity of the paint on which its working properties depend, and later determine the manner of film formation and structure. Usually they develop quickly and promote dispersion of the pigment. There are cases, however, which can be regarded as dramatic exceptions ; for example, sparks up to half an inch long accompany the attempted dispersion of titanium dioxide by grinding in tri. cresyl phosphate under electrically insulated conditions, whereas in the presence of a trace of surfaceactive material (oleic acid) dispersion proceeds normally without the visible release of energy. When paint fails, that is, cracks and exfoliates, the shrink. age forces overcome the combined forces of cohesion (pigment/medium) and adhesion (film/support). When adhesion is good, the development of other paint defects is delayed.

Research into these matters has been conducted by the optical examination of paint sections. In order to use the electron microscope for examination of paint films it is necessary to prepare a replica of the paint surface on a material sufficiently thin to transmit the electron beam. This is done by taking a negative impression of the surface structure of the paint by the simple process of spreading methyl

* Substance of the Friday Evening Discourse entitled "Whither Paint?" delivered by Dr. L. A. Jordan at the Royal Institution on March 7. 
cellulose solution upon it. When dry, this is stripped off and a positive impression is prepared by allowing evaporated silica to condense on the surface in a vacuum; after removal of the methyl cellulose, the siliceous replica is ready for examination. By this technique it is possible to observe the disiuption of a paint surface in its early stages-even after a few hours of exposure to accelerated ageing and weathering in the laboratory.

Red lead is generally still the first choice as a rustinhibitive pigment in spite of many interesting developments in the study of corrosion of iron. Its disadvantages are its density and scarcity. Lead cyanamide is of lighter colour and somewhat lower density, and is being developed as a substitute for red lead.

During the War many special-purpose paints were used; for example, luminous paints, conducting paints and paints sensitive to changes of humidity and temperature. The active agent in almost every case was the pigment, which itself acted as the indicator of the changes, usually by a variation of colour. An interesting feature of luminous materials is that the energy stored as a consequence of activation is quickly released when the paint is heated. This energy can also be released by increasing the internal friction. Thus, when a luminous paint film is bent inwards there is a release of energy as a flash of light. When the bend is outward (internal friction not so great) the energy released is much smaller. The bending of a paint film also modifies its electrical conductivity. This effect is more readily observed with a carbon pigment, the electrical conductivity of which is presumed to be due to the chains of carbon particles which it contains. Bending inwards (compression) decreases the electrical resistance by bringing the carbon particles nearer together: bending outwards has the opposite effect. Graphite is lamellar in form, and its low resistance is ascribed to chains of overlapping plates lying within the paint film. But not all lamellar pigments form a conducting path, even if composed of a material of low resistance. The outstanding example is aluminium paint, which gives a bright metallic film but which, however, is non-conducting. The plates are insulated from each other by a surface film of polishing agent, usually a stearate, and a tightly held oxide layer. The polish. ing agent is responsible for the floating and leafing of the particles within the paint film, whence arises its lustre. A conducting paint film can be obtained from copper powder only when the surfaces are chemically clean and are maintained in the reduced condition.

The use of paint as a medium for employing chemical compounds that undergo colour changes at certain critical temperatures has met with a certain amount of success, and organic dyestuffs giving a colour change that is irreversible with temperature can be used to record temperatures inside machinery where the use of thermometers or thermo-couples would be impracticable. A particular example of this application is in recording whether or not a bearing has been overheating. Compounds with reversible colour changes have also been of use, and the double iodide of copper and mercury, which changes from red to black as the temperature rises above $90^{\circ} \mathrm{C}$. and gives the reverse colour change on cooling, served, during the War, as an indicator on the distillation apparatus of lifeboats. It was easier for men in distress to note a change of colour than to have to read a thermometer.
In recent years most old-established industries have attempted to introduce scientific tests that would be independent of the human element, and the paint industry is no exception. A difficulty arises, however, in the recording of such qualities of a painted surface as 'gloss' and 'lustre', which are normally judged by the human eye. Experience of instruments devised for such measurement has shown that these properties are not measurable in the same way as mass and length, but that they have a subjective element which, so far, has defied instrumental interpretation.

This brings us to the fundamental characteristic, the measurement of colour, which provides its own psychological problem. Accurate tricolorimetric or spectrophotometric measurement permits recording of primary colour and brightness values which are now accepted as the ultimate standards against which the colour patterns or secondary standards may be checked at intervals. The difficulty arises in deciding what can be taken as an acceptable match to a standard colour with permissible tolerances. The methods of colorimetry have reached a high degree of precision, but they only deal with colour objectively; they measure stimuli and not the sensation of colour. What is wanted is a relationship between stimulus and the corresponding changes in mental colour-sensation, and if such a relationship could be established it would no doubt contribute to the solution of one of the outstanding problems of the paint industry, namely, control of the drift of patterns away from their original colour, even when they are kept in the dark.

As to the future, it is certain that new products will produce new and improved paints. The corrosion problem may be expected to diminish with the use of less corrodible materials; but whatever happens in this respect there will always be the desire to provide good appearance and pleasing colours.

\section{FORTHCOMING EVENTS}

(Meetings marked with an asterisk * are open to the public)

\section{Monday, June 16}

UNIVERSITY COLLEGE, IONDON (at the Institution of Electrica Engineers, Savoy Place, Victoria Embankment, Jondon, W.C.2), at 5.30 p.m.-Prof. Kapp: "The Presentation of Technical Information", Second Series, 3 : "The Part Played by Logic in Presentation"."

\section{Tuesday, June 17}

RESEARCH DEFENCE SOCIETY (at the Royal Society of Tropical Medicine and Hygiene, Manson House, 26 Portland Place, London, W.1), at 3.15 p.m.- Annual General Meeting. Prof. G. H. Wooldridge : "What Animals Owe to Experimental Research" (Sixteenth Stephen Paget Memorial Lecture).

MEDICAL RESEARCH CoUNCIL (at the Royal Institution, 21 Albemarle Street, London, W. 1), at 5 p.m.-Dr. J. G. Hamilton (Radiation in Medicine"."*

\section{Wednesday, June 18}

Geological Societr (at Burlington House, Piccadilly, London, W.1), at 5 p.m.-Mr. C. E. N. Bromehead: "A Geological Museum of the Early Seventeenth Century".

ROYAX METEOROLOGICAL SOCIETY (at 49 Cromwell Road, London, S.W.7), at 5 p.m.- Sir Gilbert T. Walker. F.R.S.: "Arctic Conditions and World Weather"; Mr. S. Duvdevani: "An Optical Method of Dew Estimation".

\section{Thursday, June 19}

Chadwick Public Lecture (at the Chelsea Physic Garden, Swan Walk, London, S.W.3), at 4 p.m.- Prof. W. H. Pearsall, F.R.S.: "Water Supply and Freshwater Biology"."

ROYAL SOCIETy (at Burlington House, Piccadilly, London, W.1), at 4.30 p.m.-Mr. B. M. Cwilong: "Sublimation in a Wilson, Chamber"; Sir Lawrence Bragg, F.R.S., and Mr. J. F. Nye: "A Dynamical Model of a Crystal Structure". 